\title{
Surgery modifies cardiac sensory transduction
}

\author{
Mathias Waldmann, MD \\ Robert P-C. Chen, MD ${ }^{\mathrm{b}}$ \\ J. Andrew Armour, MD, $\mathrm{PhD}^{\mathrm{c}}$
}

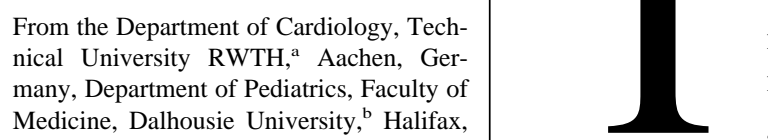

Objective: We sought to determine whether cardiac surgery, specifically the placement of an incision within a ventricular wall, affects the capacity of regional cardiac sensory nerve terminals (neurites) to transduce the local cardiac milieu.

Methods: The capacity of sensory neurites in the right ventricular outflow tract associated with afferent neurons in nodose ganglia to transduce their mechanical and chemical milieu was studied in 11 anesthetized pigs before and after performing a local ventriculotomy.

Results: Right ventricular outflow tract sensory neurites associated with 23 nodose ganglion afferent neurons were identified that transduced local mechanical deformation along with substance P. The capacity of these sensory neurites to transduce these stimuli was almost totally obtunded after local ventriculotomy.

Conclusions: The capacity of afferent neurons to transduce the cardiac milieu can be modified by cardiac surgical interventions. This may have negative implications with respect to how the entire cardiac neuronal hierarchy transduces alterations in the cardiac milieu postsurgery. This fact should be taken into account when contemplating the placement of cardiac incisions during surgery.

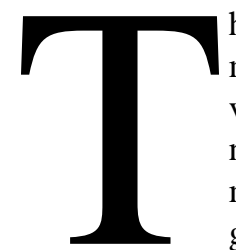

he cardiac milieu is transduced to second order neurons in the medulla and spinal cord through sensory nerve endings (neurites) in various regions of the heart that are associated with somata in nodose $^{1-3}$ and dorsal root $^{4}$ ganglia, respectively. In anesthetized mammals, most cardiac sensory neurites associated with nodose ganglion somata generate activity that is random in nature and of low Nova Scotia, Canada, and Department of Pharmacology, Faculty of Medicine, University de Montréal, ${ }^{\mathrm{c}}$ Montréal, Québec, Canada.

This work was supported by a grant from the Canadian Institutes of Health Research, the Nova Scotia Heart and Stroke Foundation, and the Isaac Walton Killam Research Foundation.

Received for publication June 24, 2002; revisions requested Aug 26, 2002; revisions received Oct 21, 2002; accepted for publication Jan 14, 2003.

Address for reprints: J. A. Armour, MD, $\mathrm{PhD}$, Department of Pharmacology, Faculty of Medicine, Université de Montréal, Montréal, Québec, Canada, H4J 1C5 (Email: JA-Armour@crhsc.ca).

J Thorac Cardiovasc Surg 2003;126:1792-7

Copyright (C) 2003 by The American Association for Thoracic Surgery

$0022-5223 / 2003 \$ 30.00+0$

doi:10.1016/S0022-5223(03)01038-9 with nodose ganglion afferent neurons transduce chemicals such as the peptides bradykinin and substance $\mathrm{P},{ }^{3}$ both of which are known to be liberated by the ischemic myocardium. ${ }^{6,7}$ They also transduce local deformation. ${ }^{1}$

It has been proposed that the proper transduction of the cardiac milieu by the cardiac neuronal hierarchy is fundamental to adequate functioning of the cardiac efferent nervous system. ${ }^{4}$ For instance, alterations in afferent neuronal inputs to the cardiac neuronal hierarchy may result in excessive activation of specific populations of intrinsic cardiac efferent neurons that can lead to the genesis of ventricular arrhythmias. ${ }^{8}$ Indeed, it has been reported that cardiac surgical interventions can modify the cardiac neuronal hierarchy and as a consequence influence cardiac arrhythmia formation in the postoperative period. ${ }^{9,10}$ It remains to be established whether cardiac surgery modifies the capacity of the cardiac neuronal hierarchy to transduce the mechanical or chemical milieu of the heart.

Extracellular activity generated by the somata of individual cardiac afferent neurons in nodose ganglia can be recorded for relatively prolonged periods of time using tungsten microelectrodes in situ. ${ }^{1,5}$ This experimental design permits an assessment of the response characteristics of individual cardiac afferent neurons to a variety of mechanical and chemical stimuli applied locally to their ventricular sensory fields over time, without affecting identified somata directly. By using this 
technique, we determined whether the transduction capabilities of ventricular sensory neurites associated with nodose ganglion afferent neurons are modified during the course of surgery (ie, by local ventriculotomies).

\section{Material and Methods Subjects}

Neonatal piglets ( $\mathrm{n}=11$ pigs), of either sex and weighing between 21 and $30 \mathrm{~kg}$, were used for this study. These experiments were performed in accordance with the National Institutes of Health "Guide for the Care and Use of Laboratory Animals" (National Academy Press, Washington, DC, 1996) and the Canadian Council on Animal Care. The committee for animal care and use of Dalhousie University approved this study.

\section{Animal Preparation}

Each pig was sedated with ketamine hydrochloride $(18 \mathrm{mg} / \mathrm{kg}$, intramuscularly). After endotracheal intubation, anesthesia was instigated with $4 \%$ halothane. Positive-pressure ventilation (15-20 breaths/min using $100 \% \mathrm{O}_{2}$, with a peak inspiratory pressure of 15 to $20 \mathrm{~mm} \mathrm{Hg}$ delivered by a Bird Mark 7A ventilator; Bird Products Corp, Palm Springs, Calif) was initiated, and sodium pentothal (20 mg/kg intravenous [IV] loading dose followed by 10 $\mathrm{mg} / \mathrm{kg}$ IV boluses) was administered. A midline incision was made in the ventral neck to expose the right or left nodose ganglion. Thereafter, a midline sternotomy was performed to expose the pericardium. Heart rate (identified by a lead II electrocardiogram) and other cardiovascular indices were monitored throughout the period of investigation for responses to noxious stimuli to ensure that adequate anesthesia was provided. After the surgery was completed, $\alpha$-chloralose was administered as a bolus $(25 \mathrm{mg} / \mathrm{kg}$ IV), followed by repeat doses (10-15 mg/kg IV, as required). Body temperature was maintained constant by means of a circulating hot water pad.

\section{Surgical Intervention}

A 4-cm-long incision was made along the middle of the outflow tract of the right ventricle. It began immediately inferior to the pulmonary valve and extended throughout the length of the conus to the right ventricular infundibulum. The depth of this incision included the epicardium and myocardium, but not the endocardium in most instances. This avoided the risk of significant hemorrhage. The incision was then closed with interrupted sutures (3-0 silk) to eliminate local sources of bleeding. This procedure mimicked the incision commonly used for pediatric right ventricular outflow tract surgery. Reapplication of the mechanical and chemical stimuli to right ventricular outflow tract sensory fields of identified afferent neurons was performed approximately 30 minutes after this surgical intervention was completed.

\section{Recording Cardiovascular Variables}

Left ventricular chamber pressure was measured with a $3 F$ Cordis pigtail catheter (Cordis Corp, Miami, Fla) advanced retrogradely into that chamber through a femoral artery. Systemic arterial pressure was measured with a $4 \mathrm{~F}$ Cordis catheter placed in the descending aorta through the other femoral artery. A 4F Berman angiographic catheter (Arrow International Inc, Reading, Pa) was inserted through a femoral vein into the right ventricular chamber.
These catheters were attached to Bentley Trantec model 800 pressure transducers (Bentley Trantec Inc, Irvine, Calif). All data, including the lead II electrocardiogram, were recorded on an Astro-Med model MT 9500 8-channel rectilinear recorder (AstroMed, West Warwick, RI). Data were stored on VHS tapes using a videocassette recorder (Model 820, AR Vetter Co, Rebersburg, Pa) for later analysis.

\section{Recording Afferent Neuronal Activity}

A right or left nodose ganglion was exposed through a midline incision made in the ventral skin of the neck. The ganglion was left bound to adjacent connective tissue to minimize its motion. It was covered with mineral oil to prevent its desiccation. Extracellular action potentials generated by nodose ganglion afferent neurons were recorded using a tungsten microelectrode (Model ME 2510-2, Frederick Haer \& Co, Bowdoninham, Me), as described previously. ${ }^{3,5}$ The tip of the tungsten microelectrode, with a diameter of $100 \mu \mathrm{m}$, an exposed tip of $50 \mu \mathrm{m}$, and an impedance of 9 to $12 \mathrm{MOhms}$ at $1 \mathrm{kHz}$, was positioned in the ganglion at varying depths using a micromanipulator. Reference and ground electrodes were attached separately to adjacent tissues. Signals generated by nodose ganglion afferent neurons were differentially amplified by a Princeton Applied Research (Princeton, NJ) Model 113 amplifier, with bandpass filters set at $300 \mathrm{~Hz}$ to $10 \mathrm{kHz}$ and an amplification range of 100 to 500 times. The output of this device was further amplified (50-200 times) and filtered (bandwidth: $100 \mathrm{~Hz}-2$ $\mathrm{kHz}$ ) with an optically isolated amplifier (Applied Microelectronics Institute, Halifax, NS, Canada). Its signals were led to a Nicolet Model 207 oscilloscope (Thermo Nicolet Corp, Madison, Wis) and to a Grass AM8 Audio Monitor (Grass Instruments, Quincy, Mass).

The nodose ganglion contains the somata of afferent neurons associated with sensory neurites in tissues other than the heart. An identified neuron was considered to be associated with cardiac sensory neurites when local epicardial mechanical or chemical stimuli modified the activity generated by that neuron. Individual action potentials with signal-to-noise ratios greater than $3: 1$ were identified by their amplitude and configuration. One or 2 action potentials so identified at each active locus remained similar in their amplitude and configuration over hours of recording, presumably because of a lack of motion of investigated ganglia. When the electrode was moved a few micrometers away from an active site, action potentials changed in size, not configuration. When the electrode penetrated the adjacent vagus nerve, action potentials with signals of the same amplitude as the background noise were identified. In other words, action potentials generated by individual axons of passage could not be differentiated using this methodology. At the end of the experiments, electrical stimuli $(0.1 \mathrm{~ms} ; 0.1$ $\mathrm{Hz} ; 2 \mathrm{~V}$ ) were delivered through a ball electrode to identified epicardial sensory fields (the other electrode being attached to the pericardium) to activate identified neurons. Once the latency of neuronal activation was recorded, the distance between the stimulating and recording electrodes was determined to estimate the conduction velocities of the axons associated with identified afferent neuronal somata.

\section{Mechanical Stimuli}

After a median sternotomy to expose the thoracic contents, the ventral pericardium was incised and retracted laterally to expose 
the heart. Thereafter, loci on the ventral, epicardial surface of the right ventricular outflow tract were touched gently with a salinesoaked cotton swab to identify nodose ganglion afferent neurons that transduced mechanical perturbations in that region of the heart. The right ventricular conus epicardium was chosen for investigation because it is known to contain a relatively sizable population of sensory neurites associated with nodose ganglion afferent neurons. ${ }^{4}$ The epicardial extent of each identified mechanosensory field was determined in this manner. This mechanical intervention was performed at least twice to confirm the reproducibility of its effects. Then, the superior vena cava was occluded for 3 to 5 seconds.

\section{Chemical Stimuli}

Thereafter, individual chemicals were applied for 45- to 60-second periods to identified right ventricular epicardial loci using pledgets $(1 \mathrm{~cm} \times 1 \mathrm{~cm})$ soaked with $0.5 \mathrm{~mL}$ of each respective chemical. Sensory fields were washed for 30 seconds with normal saline $(\sim 2$ $\mathrm{mL} / \mathrm{s}$ ) after each chemical-soaked pledget was removed. At least 10 minutes elapsed between these interventions. Epicardial sensory field application of pledgets soaked with room temperature normal saline ensured that vehicle or mechanical effects of pledgets did not cause observed neuronal responses.

Chemicals, obtained from Sigma Chemical Co (St Louis, Mo), were dissolved in room temperature normal saline. The agents applied to epicardial loci were the purinergic agent adenosine (10 $\mu \mathrm{mol} / \mathrm{L})$, the peptides bradykinin $(10 \mu \mathrm{mol} / \mathrm{L})$ and substance $\mathrm{P}(10$ $\mu \mathrm{mol} / \mathrm{L})$, and the sodium channel modifier veratridine $\left(5 \times 10^{-6}\right.$ $\mathrm{g} / \mathrm{cc}$ ). Each of these chemicals is known to modify the ventricular sensory neurites associated with nodose ganglion afferent neurons. ${ }^{3}$ The order of epicardial application of each chemical varied among experiments. When found to be responsive, chemicals were reapplied to the same epicardial locus to verify reproducibility of responses. The dose of each agent studied was similar to that previously established to modify cardiac sensory neurites associated with porcine neonatal nodose ganglion afferent neurons. ${ }^{5}$ At least 10 minutes elapsed between epicardial applications of each chemical such that monitored indices returned to baseline values before the next intervention. Then, these pharmacologic agents were also administered IV in the same doses in $0.1 \mathrm{cc}$ volumes to assess whether they affected monitored cardiac variables or cardiac afferent neurons if they entered the circulation in sufficient doses to do so.

\section{Data Analysis}

Heart rate, right and left ventricular systolic pressures, and aortic pressure were measured for 30 seconds before each intervention and during maximal responses elicited by each intervention. Spontaneous fluctuations of hemodynamics were minimal during control periods. Heart rate variability was less than 5 beats/min, and systolic pressure variability was less than $5 \mathrm{~mm} \mathrm{Hg}$ during control conditions. Action potentials generated by identified nodose ganglion neurons were counted during 60-second periods immediately before a stimulus was applied and when responses to a stimulus had reached steady state. Data are expressed as the mean \pm SEM. Neuronal activity data are expressed as absolute change in activity from baseline. This was done because cardiac sensory neurons respond to similar interventions by increasing activity when rela- tively quiescent or decreasing activity when tonically active. ${ }^{3}$ Thus, when the activity generated by individual afferent neurons changed in response to an intervention, the total change in afferent neuronal activity from baseline values was assessed. ${ }^{3,5}$ Data derived before and during each intervention were compared using 2 -way analysis of variance. Significance was assigned at a $P$ value less than .01 .

\section{Results \\ Mechanical Stimuli}

The activity generated by identified cardiac afferent neurons in right- or left-sided nodose ganglia was similar and did not correlate with cardiac or respiratory mechanics. The activity generated by nodose ganglion afferent neurons associated with sensory neurites identified in the epicardial outflow tract of the right ventricle of each pig changed when local mechanical stimuli were applied to their sensory fields (Table 1). In 8 of the pigs, the spontaneous activity generated by their associated somata increased; in the 3 other pigs, afferent neuronal activity decreased when gentle mechanical stimuli were applied to their associated epicardial sensory neurites. Overall, afferent neuronal activity changed by $41 \pm 6$ impulses per minute in response to local epicardial deformation (Table 1). These responses were initiated immediately after the mechanical stimulus was applied and terminated on its removal. Repeat application of mechanical stimuli to an identified epicardial sensory field induced similar afferent neuronal responses. Studied afferent neurons did not respond to mechanical stimuli applied to pulmonary or vascular tissues or, for that matter, other regions of the heart. At the end of the experiments, electrical stimulation $(1 \mathrm{~V} ; 0.1 \mathrm{~ms} ; 0.1 \mathrm{~Hz})$ applied to axons craniad to the incision site was performed to activate identified somata. The conduction velocities of axons associated with identified neurons were estimated to be, on average, $2.7 \pm 0.9 \mathrm{~ms}$.

\section{Chemical Stimuli}

Substance $\mathrm{P}$ elicited the greatest change in afferent neuronal activity, modifying the activity generated by identified afferent neurons in all 11 animals (77 $\pm 16 \mathrm{impulses} / \mathrm{min}$ ). Afferent neuronal activity increased in 8 animals and decreased in 3 animals when substance $P$ was applied to the epicardium of the right ventricular outflow tract. The afferent neuronal activity was changed by veratridine in 6 animals, by bradykinin in 4 animals, and by adenosine in 2 animals (Table 1).

Responses began within 10 to 30 seconds of chemical application, lasting for up 2 minutes after removal and flushing of the epicardial site with saline. Repeat application of an active chemical to an identified epicardial sensory field induced similar neuronal activity responses. Monitored cardiovascular indices were affected when substance $\mathrm{P}$ was applied to epicardial loci in 2 animals (Figure 2). In control states, the spontaneous activity generated by identified car- 


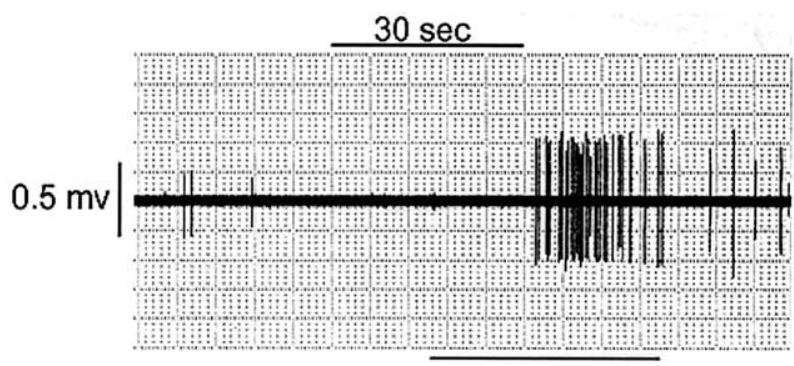

Figure 1. When a ventriculotomy (longitudinal cut in the right ventricular conus; performed at the time indicated by the bar below) was performed in a pig, a burst of activity was generated by 2 nodose ganglion afferent neurons.

diac afferent neurons was not affected when saline-soaked pledgets were applied to their associated epicardial sensory fields. Systemic administration of each chemical in the doses tested did not alter the activity generated by identified cardiac afferent neurons or recorded cardiovascular variables.

\section{Ventriculotomy}

In 2 instances, identified afferent neurons were activated as the incision was made along the right ventricular outflow tract (Figure 1). After these incisions were performed, the activity generated by most neurons was reduced (Figure 2), 2 becoming silent thereafter. The activity generated by identified afferent neurons remained unresponsive to mechanical and chemical stimuli applied to the same right ventricular outflow tract loci after cutting the midline of the ventral surface of the right ventricular outflow tract (Table 1). This lack of afferent neuronal responsiveness occurred despite the fact that adjacent nodose ganglion afferent neurons with sensory neurites in other ventricular regions were capable of responding to alterations in the mechanical milieu of the heart induced by brief occlusion of the superior vena cava.

\section{Discussion}

These data indicate that the capacity of ventricular sensory neurites associated with afferent somata in nodose ganglia to transduce the mechanical and chemical milieu of the heart can remodel after cardiac surgery, depending on the sensory fields affected by the incision. This finding may have relevance with respect to the suggestion that cardiac surgical incisions can compromise the proper functioning of the cardiac neuronal hierarchy such that the postoperative heart becomes predisposed to arrhythmia formation. ${ }^{10}$

Ventricular sensory neurites associated with afferent neurons in porcine nodose ganglia transduce mechanical and multiple chemical stimuli. ${ }^{5}$ All cardiac afferent neurons identified in this study proved to be sensitive to mechanical and chemical stimuli. Afferent neuronal activity increased
TABLE 1. Change in nodose ganglion afferent neuronal activity (reported as average \pm SEM) induced in all $\mathbf{1 1}$ preparations during application of mechanical or chemical stimuli to their associated right ventricular outflow tract sensory neurites, as recorded before and after performing a ventriculotomy through their sensory fields

\begin{tabular}{|c|c|}
\hline $\begin{array}{l}\text { Intervention } \\
\text { (n = } 11 \text { animals) }\end{array}$ & $\begin{array}{c}\text { Change in } \\
\text { neuronal activity } \\
\text { (delta IPM) }\end{array}$ \\
\hline $\begin{array}{l}\text { Before incision } \\
\text { Control }(n=11)\end{array}$ & \\
\hline $\begin{array}{l}\text { Mechanical stimulus } \\
\text { Control }(\mathrm{n}=11)\end{array}$ & $41.1 \pm 6.4^{*}$ \\
\hline $\begin{array}{l}\text { Substance P } \\
\text { Control }(\mathrm{n}=4)\end{array}$ & $77.0 \pm 15.8^{*}$ \\
\hline $\begin{array}{l}\text { Bradykinin } \\
\text { Control }(\mathrm{n}=2)\end{array}$ & $29.3 \pm 16.4$ \\
\hline $\begin{array}{l}\text { Adenosine } \\
\text { Control }(n=6)\end{array}$ & $4.7 \pm 10.3$ \\
\hline $\begin{array}{l}\text { Veratridine } \\
\text { After incision } \\
\text { Control }\end{array}$ & $11.8 \pm 5.1$ \\
\hline $\begin{array}{l}\text { Mechanical stimulus } \\
\text { Control }\end{array}$ & $4.4 \pm 3.2$ \\
\hline $\begin{array}{l}\text { Substance P } \\
\text { Control }\end{array}$ & $10.0 \pm 4.9$ \\
\hline $\begin{array}{l}\text { Bradykinin } \\
\text { Control }\end{array}$ & $2.1 \pm 1.6$ \\
\hline $\begin{array}{l}\text { Adenosine } \\
\text { Control }\end{array}$ & $1.8 \pm 1.1$ \\
\hline Veratridine & $1.1 \pm 0.8$ \\
\hline
\end{tabular}

IPM, Impulses per minute.

In control states (before incision), application of gentle touch or substance $P$ to their sensory fields modified afferent neuronal activity. After performing the ventriculotomy (after incision), afferent neurons no longer responded to these local stimuli. The activity generated by afferent neurons in response to each intervention is tabulated according to change in neuronal activity from preintervention (baseline) values (delta IPM).

${ }^{*} P<.01$, comparing data obtained before and during application of local epicardial stimuli.

or decreased when their associated ventricular sensory neurites were exposed to such stimuli. Concurring with previous data, ${ }^{3,5}$ their activity was enhanced or suppressed when their associated ventricular sensory neurites were exposed to a stimulus, depending on the functional status of the afferent neuron tested. For instance, nodose ganglion afferent neurons that generated low levels of activity in control states responded when substance $\mathrm{P}$ was applied to its sensory field by increased levels of activity. On the other hand, afferent neurons generating relatively high spontaneous activity in control states were suppressed by that intervention. Such data have been interpreted as indicative of the fact that sensory transduction by individual cardiac afferent neurons is unique with respect to the various stimuli they transduce and the level of activity they generate at the time of cardiac milieu alterations. 


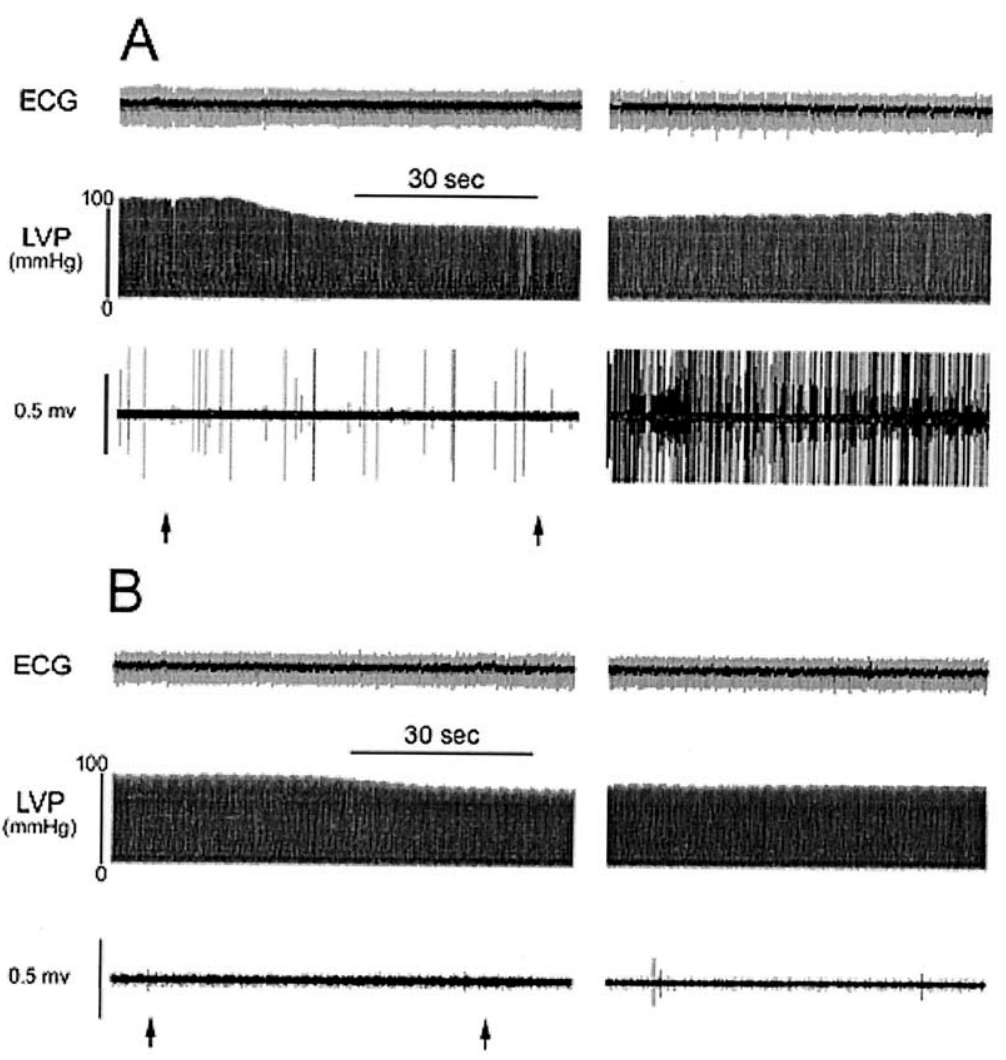

Figure 2. A, In control states after application of a pledget soaked with the peptide substance $P$ (between arrows below) to the right ventricular conus epicardium, the activity generated by a few spontaneously active afferent neurons increased (bottom trace); excitation of these afferent neurons persisted beyond the short-lived reflexinduced hypotension. B, After a ventriculotomy was performed through this sensory field, repeat application of substance $P$ to the same epicardial site (between arrows below) failed to excite these afferent neurons. This stimulus also induced modest reduction in left ventricular pressure. A record of 1-minute duration was deleted from these traces after application, as represented by the blank spaces between the panels.

That activity generated by afferent neurons was not modified when saline was applied to their sensory fields indicates that activity changes induced by epicardial application of a chemical were not caused by vehicle, temperature change, or local mechanical effects. It is unlikely that a chemical, when applied topically to a limited area of the right ventricular outflow tract in the doses studied, entered the systemic circulation in sufficient doses to directly affect cardiac myocytes or peripheral resistance vessels because monitored cardiovascular variables were not affected overall when each chemical was administered systemically in the same doses. In 2 animals, epicardial application of substance $\mathrm{P}$ resulted in left ventricular pressure modification (Figure 2). Presumably, in those instances sufficient populations of cardiac sensory neurons were excited thereby to induce reflex modification of cardiovascular variables. That such reflex responses were only partially blunted after the ventriculotomy presumably indicates that the chemical stimulus activated lesser populations of ventricular sensory neurites in such a state, and that adjacent ones were not affected by the incision.

That the capacity of cardiac afferent neurons to sense their regional mechanical and chemical milieu becomes obtunded after local ventriculotomy indicates that remodeling of sensory transduction by the cardiac neuronal hierarchy may occur after a ventriculotomy. Obviously, the degree of such remodeling would be dependent on the location and size of the ventriculotomy. The former is evident because sensory neurites associated with individual afferent neurons lie concentrated in the outflow tracts of either ventricle. ${ }^{4}$ That a surgical intervention might interfere with the transduction capabilities of cardiac sensory neurites has implications with respect to how the entire cardiac neuronal hierarchy functions after cardiac surgery.

Remodeling of cardiac sensory transduction in the immediate postoperative period presumably would affect the capacity of the cardiac neuronal hierarchy to transduce cardiac events in the maintenance of adequate cardiac ef- 
ferent neuronal function. Given the redundancy of function of the multiple sensory neurites found in cardiac regions ${ }^{4}$ and their presumed capacity to regenerate after damaging their axons, ${ }^{11}$ derangement of their capacity to transduce the cardiac milieu might not be long-lasting. Incisions made in the walls of the cardiac chambers can also involve autonomic efferent postganglionic axons. Having stated that, the degree of damage induced by local ventricular mechanical trauma needs to be extensive to obtund the capacity of the multiple populations of sympathetic efferent postganglionic axons to affect regional ventricular contractility. ${ }^{11-13}$ Given this and the fact that trauma to specific ventricular regions may not obtund the capacity of cardiac efferent neurons to affect cardiac function significantly, any remodeling of the cardiac neuronal hierarchy after cardiac surgery may primarily involve its capacity to transduce cardiac regional sensory information.

We gratefully acknowledge the technical assistance of Richard Livingston.

\section{References}

1. Armour JA, Huang MH, Pelleg A, Sylvén C. Responsiveness of in situ canine nodose ganglion afferent neurons to epicardial mechanical or chemical stimuli. Cardiovasc Res. 1994;28:1218-25.
2. Coleridge HM, Coleridge JCG, Kidd C. Cardiac receptors in the dog, with particular reference to two types of afferent endings in the ventricular wall. J Physiol (Lond). 1994;174:323-39.

3. Thompson GW, Horackova M, Armour JA. Chemotransduction properties of nodose ganglion cardiac afferent neurons in guinea pigs. Am J Physiol. 2000;279:R433-9.

4. Armour JA. The role of peripheral autonomic neurons in cardiac regulation. In: Armour JA, Ardell JL, editors. Neurocardiology. New York: Oxford University Press; 1994. pp. 219-44.

5. Chen R P-C, Thompson GW, Armour JA. Transduction capabilities of neonatal ventricular afferent neurons in situ. Auton Neurosci. 2001;87:1-8.

6. Kimura E, Hashimoto K, Furukawa S, Kayakawa H. Changes in bradykinin level in coronary sinus blood after the experimental occlusion of a coronary artery. Am Heart J. 1973;85:635-47.

7. Rubio R, Berne RM, Katori M. Release of adenosine in reactive hyperemia of the dog heart. Am J Physiol. 1962;216:56-62.

8. Armour JA. Comparative effects of endothelin and neurotensin on intrinsic cardiac neurons. Peptides. 1996;17:1047-52.

9. Bertrand ME, LaBlanche JM, Rousseau MF, Warembourg HH, Stankowtka C, Soots G. Surgical treatment of variant angina: use of plexectomy with aortocoronary bypass. Circulation. 1980;61:877-82.

10. Davis Z, Jacobs HK, Bonilla J, Anderson RA, Thomas C, Forst W. Retaining the aortic fat pad during cardiac surgery decreases postoperative atrial fibrillation. Heart Surg Forum. 2000;3:108-12.

11. Arora RC, Hirsch G, Hirsch K, Armour JA. Transmyocardial laser revascularization remodels the intrinsic cardiac nervous system in a chronic setting. Circulation. 2001;104:I101-20.

12. Johnstone D, Janes RD, Klassen GA, Armour JA. Functional integrity of sympathetic efferent postganglionic axons in a region of stunned myocardium. Can J Cardiol. 1989;5:357-64.

13. Kaye MP, Brynjolfsson GG, Geis WP. Chemical epicardiectomy. Cardiologia. 1968;53:139-49. 